\title{
Strong 0-dimensionality in Pointfree Topology
}

\author{
Bernhard Banaschewski \\ McMaster University, Hamilton, Ontario, Canada \\ iscoe@math.mcmaster.ca
}

\begin{abstract}
Classically, a Tychonoff space is called strongly 0-dimensional if its Stone-Čech compactification is 0-dimensional, and given the familiar relationship between spaces and frames it is then natural to call a completely regular frame strongly 0-dimensional if its compact completely regular coreflection is 0-dimensional (meaning: is generated by its complemented elements). Indeed, it is then seen immediately that a Tychonoff space is strongly 0-dimensional iff the frame of its open sets is strongly 0-dimensional in the present sense.

This talk will provide an account of various aspects of this notion. Particuarly relevant for this will be

-the saturation quotient $S M$ of a compact normal frame $M$, given by the saturation nucleus $s_{M}$ on $M$ for which $s_{M}(a)=\bigvee\{x \in M \mid x \vee y=e \Rightarrow a \vee y=e\}$.

-the variants of normality expressed by the following conditions: if $a \vee b=e$ (the top) then there exists $c \leq b$ such that $a \vee c=e$ for which
\end{abstract}

$$
c \prec b \text { (normal) } \quad c \prec \prec b \text { (completely normal) } \quad c \text { complemented (strongly normal) }
$$

where $c \prec b$ means $b \vee c^{*}=e$ ( $c^{*}$ the pseudocomplement of $c$ ) and $c \prec \prec b$ indicates the existence of an infinite sequence of interpolations

$$
c \prec b, c \prec d_{11} \prec b, c \prec d_{21} \prec d_{11} \prec d_{23} \prec b, \ldots
$$

-the cozero part Coz $L$ of a completely regular frame $L$ consisting of all elements $\operatorname{coz}(\gamma)=\gamma((-, 0) \vee(0,-))$ for the real-valued continuous functions $\gamma$ on $L$ (corresponding to the classical cozero sets of a space), and

-a representation of the compact completely regular coreflection of a completely regular frame $L$ as

$$
S \mathcal{J C o z} L \rightarrow L, I \mapsto \bigvee\{\operatorname{coz}(\gamma) \mid \operatorname{coz}(\gamma) \in I\}
$$

where $\mathcal{J} \operatorname{Coz} L$ is the frame of ideals of the lattice $\operatorname{Coz} L$.

The latter will be used to obtain the following pointfree form of a classical result: A completely regular frame $L$ is strongly 0-dimensional iff every cozero elements of $L$ is a countable join of complemented ones.

Further, it will be shown that some familiar types of completely regular frames are strongly 0dimensional and characterized by very natural additional conditions. This will include the $P$-frames, that is, the completely regular frames all whose cozero elements are complemented; they turn out to be the strongly 0-dimensional frames in which any countable join of complemented elements is complemented. The latter are called the $P_{0}$-frames, and it will be shown that they are reflective in the category of all 0-dimensional frames. This parallels the corresponding result for $P$-frames in relation to all completely regular frames, but with a substantially simpler proof. It remains a challenging open question whether $P_{0}=P$. 